Accepted by The Astrophysical Journal (2008, Mar) [AStro-Ph version w/LOW-Resolution images]

Preprint typeset using $\mathrm{LAT}_{\mathrm{E}} \mathrm{X}$ style emulateapj v. 03/07/07

\title{
STAR FORMATION IN THE MOST DISTANT MOLECULAR CLOUD IN THE EXTREME OUTER GALAXY: A LABORATORY OF STAR FORMATION IN AN EARLY EPOCH OF THE GALAXY'S FORMATION ${ }^{1,2,3}$
}

\author{
NaOto Kobayashi ${ }^{4,5}$, Chikako Yasui \\ Institute of Astronomy, School of Science, University of Tokyo, 2-21-1 Osawa, Mitaka, Tokyo 181-0015, Japan
}

Alan T. TOKUnAgA ${ }^{4}$

Institute for Astronomy, University of Hawaii, 2680 Woodlawn Drive, Honolulu, HI 96822

AND

Masao Saito

ALMA Project, National Astronomical Observatory of Japan, 2-21-1 Osawa, Mitaka, Tokyo 181-8588, Japan Accepted by The Astrophysical Journal (2008, Mar) [astro-ph version w/low-resolution images]

\section{ABSTRACT}

We report the discovery of active star formation in Digel's Cloud 2, which is one of the most distant giant molecular clouds known in the extreme outer Galaxy (EOG). At the probable Galactic radius of $\sim 20 \mathrm{kpc}$, Cloud 2 has a quite different environment from that in the solar neighborhood, including lower metallicity, much lower gas density, and small or no perturbation from spiral arms. With new wide-field near-infrared (NIR) imaging that covers the entire Cloud 2, we discovered two young embedded star clusters located in the two dense cores of the cloud. Using our NIR and ${ }^{12} \mathrm{CO}$ data as well as H I, radio continuum, and IRAS data in the archives, we discuss the detailed star formation processes in this unique environment. We show clear evidences of a sequential star formation triggered by the nearby huge supernova remnant, GSH 138-01-94. The two embedded clusters show a distinct morphology difference: the one in the northern molecular cloud core is a loose association with isolated-mode star formation, while the other in the southern molecular cloud core is a dense cluster with cluster-mode star formation. We propose that high compression by the combination of the SNR shell and an adjacent shell caused the dense cluster formation in the southern core. Along with the low metallicity range of the EOG, we suggest that EOG could be an excellent laboratory for the study of star formation processes, such as those triggered by supernovae, that occured during an early epoch of the Galaxy's formation. In particular, the study of the EOG may shed light on the origin and role of the thick disk, whose metallicity range matches with that of the EOG well.

Subject headings: infrared: stars — stars: formation — stars: pre-main-sequence - open clusters and associations: general — supernova remnants — ISM: clouds — Galaxy: disk — Galaxy: formation

\section{INTRODUCTION}

The extreme outer Galaxy (EOG), which we define as the region with a Galactic radius $\left(R_{\mathrm{g}}\right)$ of more than 18 kpc, has a very different environment from the regions near our solar system, since it has a much lower gas density, lower metallicity (see the metallicity gradient curve in, e.g., Smartt \& Rolleston 1997) and small or no perturbation from spiral arms. Such a region is not only of strong interest in itself, but it also provides a good opportunity to study astronomical processes, such as star formation, in a physical environment that is very differ-

\footnotetext{
Electronic address: naoto@ioa.s.u-tokyo.ac.jp

1 Based on data collected at University of Hawaii $2.2 \mathrm{~m}$ telescope.

2 This research has made use of the NASA/IPAC Infrared Science Archive, which is operated by the Jet Propulsion Laboratory, California Institute of Technology, under contract with the $\mathrm{Na}$ tional Aeronautics and Space Administration.

3 This research used the facilities of the Canadian Astronomy Data Centre operated by the National Research Council of Canada with the support of the Canadian Space Agency.

${ }^{4}$ Visiting Astronomer, University of Hawaii $2.2 \mathrm{~m}$ Telescope.

${ }^{5}$ Half of the work was done when visiting at the University of Hawaii during 1996-1999.
}

ent from that of the solar neighborhood. While the detailed star formation processes have been studied mostly for nearby star-forming regions at the distances less than $1 \mathrm{kpc}$, where the physical/chemical environment appears to be relatively uniform, the EOG enables us to study how the environment (density, temperature, pressure, external radiation field, metallicity, etc.) affects the basic star formation processes and parameters such as the initial mass function, star formation efficiency, and disk formation efficiency.

Wouterloot \& Brand (1989), Wouterloot et al. (1990), Brand \& Wouterloot (1995) obtained the first census of molecular clouds in the outer Galaxy based on the CO survey of IRAS point sources. Digel, de Geus, \& Thaddeus (1994) found eleven molecular clouds, including Cloud 2, in the EOG based on $\mathrm{CO}$ observations of distant H I peaks in the Maryland-Green Bank survey (Westerhout \& Wendlant 1982). Following those pioneering works, Heyer et al. (1998) conducted a comprehensive CO survey of molecular clouds in the outer Galaxy with the Five College Radio Astronomy Observatory (FCRAO) CO survey. Recently, Ruffle et al. (2007) conducted a molecular line survey of some of the Di- 
Kobayashi et al.

gel clouds and provided maps and chemical modeling of Cloud 2. Such detailed study will eventually reveal how those molecular clouds were formed in the EOG. As for the star formation activity, de Geus et al. (1993) first found an H II region associated with Digel's Cloud 2. Later Kobayashi \& Tokunaga (2000) found associated red infrared sources to confirm the star-forming activity in this cloud. Santos et al. (2000) and later Snell, Carpenter, \& Heyer (2002) found a considerable number of embedded star clusters in molecular clouds in the outer Galaxy that confirm star formation in clusters is common in the outer Galaxy up to $R_{\mathrm{g}} \sim 17 \mathrm{kpc}$. Recently, Brand \& Wouterloot (2007) also reported a discovery of an embedded cluster in a molecular cloud, WB89-789, at the probable Galactic radius of $\sim 20 \mathrm{kpc}$, which marks one of the most distant embedded clusters in the EOG.

Here we report the discovery of two embedded clusters in Digel's Cloud 2, which is one of the most distant molecular clouds in the EOG (Digel et al. 1994). While conducting a near-infrared survey of the Digel clouds to study the star formation activity in the EOG (Kobayashi \& Tokunaga 2001), we found clear evidence of star formation activity in Cloud 2 (Kobayashi \& Tokunaga 2000). As a follow-up study, we are extensively studying this cloud with deep near-infrared imaging using the Subaru $8.2 \mathrm{~m}$ telescope (Yasui et al. 2006, 2008) and with CO observations using the Nobeyama $45 \mathrm{~m}$ telescope (M. Saito et al. 2008, in preparation).

Originally, the kinematic distance to Cloud 2 was estimated at $R_{\mathrm{g}}=28 \mathrm{kpc}$ (heliocentric distance: $D=21$ kpc), while the latest H I observation by Stil \& Irwin (2001) suggests $R_{\mathrm{g}}=23.6 \mathrm{kpc}(D=16.6 \mathrm{kpc})$. Ruffle et al. (2007) use $R_{\mathrm{g}}=22$ and $28 \mathrm{kpc}$ for their distance sensitive calculations. However, several authors questioned this rather high $R_{\mathrm{g}}$ from independent distance estimates of the associated early-type star, MR-1 (Muzzio \& Rydgren 1974). The association of MR-1 with Cloud 2 appears to be quite firm based on the association of the $\mathrm{H} \alpha$ nebulae (de Geus et al. 1993) and the photodissociation region (Kobayashi \& Tokunaga 2000), and because all three components have almost the same LSR velocity: $-102.4 \mathrm{~km} \mathrm{~s}^{-1}$ for Cloud 2 (Digel et al. 1994); -102.7 $\pm 12 \mathrm{~km} \mathrm{~s}^{-1}$ for MR-1 (Russeil, Adami, \& Georgelin 2007) and $-101 \mathrm{~km} \mathrm{~s}^{-1}$ for the $\mathrm{H} \alpha$ nebulae (de Geus et al. 1993). Smartt, Dufton, \& Rolleston (1996) estimated the Galactic radius of MR-1 as $R_{\mathrm{g}}=15-19 \mathrm{kpc}$ $(D=8-12 \mathrm{kpc})$ with high-resolution optical spectra: the shorter and the longer distances are based on LTE and non-LTE model stellar atmospheres. Recently, Russeil et al. (2007) re-estimated the distance to MR-1 as $R_{\mathrm{g}}=14.3 \pm 0.5 \mathrm{kpc}(D=6.78 \pm 0.59 \mathrm{kpc})$ using a newly obtained high-resolution optical spectrum and an LTE model stellar atmosphere, which is consistent with the LTE distance $(D=8 \mathrm{kpc})$ by Smartt et al. (1996). Because the spectroscopic distance of stars should be more accurate than kinematic distance, the actual distance to Cloud 2 is likely to be that of MR-1, which is less than the kinematic distance calculated from $\mathrm{H} \mathrm{I} / \mathrm{CO}$ data. Throughout this paper, we adopt the most likely distance $R_{\mathrm{g}}=19 \mathrm{kpc}(D=12 \mathrm{kpc})$ for Cloud 2 , the same one used in our previous paper (Kobayashi \& Tokunaga 2000) because a non-LTE model is more likely to be accurate for stars in the effective temperature regime of MR-1 (Smartt et al. 1996). It is also the largest distance among those estimated by stellar spectroscopy, and more consistent with the radio kinematic distances. When we discuss about distance sensitive parameters, we will mention the possible systematic uncertainty.

The ambient H I density at $R_{\mathrm{g}} \sim 20 \mathrm{kpc}$ is thought to be very low because of the small surface density and the large-scale height: $N_{\mathrm{HI}}$ could be as low as $0.001 \mathrm{~cm}^{-3}$ (e.g., Nakanishi \& Sofue 2003). The metallicity of Cloud 2 is estimated at -0.7 dex from the radio molecular lines (Lubowich et al. 2004; see also Ruffle et al. 2005), which is consistent with the metallicity of MR-1 as measured by optical spectroscopy $(-0.5$ to -0.8 dex: Smartt et al. 1996; Rolleston et al. 2000).

The latest H I observation by Stil \& Irwin (2001) revealed that a remarkably large supernovae remnant (SNR) shell, GSH 138-01-94, is associated with Cloud 2. Because there is little or no perturbation from the spiral arms in the EOG enviroment, the supernova (SN) could have triggered star formation in Cloud 2. It is usually hard to determine what triggers star formation in the inner part of the Galactic disk because of many chance projections of various foreground and background objects and because the observed star-forming region itself has a complex structure of gas/dust and stars from its long star formation history and high ambient gas density. In such a region, a SNR shell cannot keep its uniform shape for a long enough time (e.g., a few Myr) to discern the SN-triggered star formation process because of the large amount of ambient material distributed nonuniformly. However, the SNR shell expansion can be clearly observed in the EOG, which is free from the above complexity. Actually, GSH 138-01-94 shows a large complete SNR shell that has lasted for more than $4 \mathrm{Myr}$, and is the largest and oldest SNR known in the Galaxy (Stil \& Irwin 2001). Therefore, Cloud 2 is an excellent place to study SN-triggered star formation, which is thought to be one of the major star formation processes in galaxies (e.g., Elmegreen 2002).

The low metallicities in the EOG regions are comparable to those for Galactic thick disk stars (e.g., Brewer \& Carney 2006), dwarf irregular galaxies, and damped Lyman- $\alpha$ systems (e.g., Pettini 2004). In conjunction with the very low gas density and lack of spiral arm perturbation, the EOG may approximate the environment of star formation in the early universe. Because dwarf irregular galaxies have a similar environmentlow-metallicity, low-gas density, and lack of spiral arm perturbation, they are also thought to represent the conditions of star formation in the early universe (e.g., Hunter, Elmegreen, \& Martin 2006). However, the EOG is advantageous for the study of such star formation processes simply because of its proximity compared to the galaxies in the local univserse. Studies of the chemical composition of halo stars in our Galaxy show that star formation in the early epoch of the Galaxy's formation was mostly triggered by supernovae (Ryan, Norris, \& Beers 1996; Audouze \& Silk 1995; Shigeyama \& Tsujimoto 1998). Therefore, Cloud 2 and other EOG star-forming regions are excellent laboratories in which to study the star formation process in an environment that is similar to that which existed during an early epoch of the Galaxy's formation.

In this paper, we discuss our near-infrared as well as ${ }^{12} \mathrm{CO}$ observational results for Cloud 2 in the above con- 
text. We also make use of the mid-infrared (IRAS), H I, and radio continuum data in the archives for our discussion. The companion papers by Yasui et al. $(2006,2008)$ discuss very deep infrared imaging of the star-forming clusters with the Subaru $8.2 \mathrm{~m}$ telescope, and they reinforce our interpretation.

\section{NEAR-INFRARED IMAGING}

Near-infrared images of Cloud 2 in the standard $J_{-}, H$-, and $K$-band filters were obtained over a five-night period (3-7 October 1998) using the QUick InfRared Camera (QUIRC) at the University of Hawaii $2.2 \mathrm{~m}$ telescope on Mauna Kea. QUIRC uses a $1024 \times 1024 \mathrm{HgCdTe}$ Astronomical Wide Area Infrared Imaging (HAWAII) array and was used at the $\mathrm{f} / 10$ focus to provide a plate scale of $0{ }^{\prime \prime} 1886$ pixel $^{-1}$ with a field of view of roughly $3 ! 2 \times$ 3.2 . The entire arc-shaped Cloud 2 was covered with a mosaic of QUIRC fields. For the present paper, we used only the dataset of the third night, when the seeing conditions were best $(\sim 0 \prime \prime 5)$ and the star clusters were clearly resolved. The sky was covered with occasional thin cirrus on that night, but the photometric uncertainty due to cirrus was insignificant. The total integration time was $5,10,15$ min for $K$-, $H$-, and $J$-bands, respectively.

All the data for each band were reduced with IRAF ${ }^{6}$ with standard procedures: dark subtraction, flat-fielding, bad-pixel correction, median-sky subtraction, image shifts with dithering offsets, and combining. The images of eight-fields were combined into one large mosaic with standard IRAF image matching tasks.

Figure 1 shows a $J H K$ three-color image of Cloud 2 with ${ }^{12} \mathrm{CO}(1-0)$ contours overplotted (the radio data were newly obtained with Nobeyama $45 \mathrm{~m}$ telescope). Locations of bright near-infrared (NIR) sources identified by Kobayashi \& Tokunaga (2000) and MR-1 (Muzzio \& Rydgren 1974) are also indicated. In this infrared color image, two clusters of red sources (Cloud 2-N cluster, Cloud 2-S cluster) were identified in the two $\mathrm{CO}$ cores of the molecular cloud (Cloud 2-N and Cloud 2S). ${ }^{7}$ The Cloud 2-N cluster was first discovered with the QUIRC image, while the Cloud 2-S cluster was already recognized as an infrared source, IRS 2, in Kobayashi \& Tokunaga (2000) and was resolved as a star cluster in the QUIRC image.

We performed photometry of those red sources and found that many of them have $H-K$ color excess in the $J-H$ vs. $H-K$ color-color diagram. We present the photometric properties of those red sources in companion papers by Yasui et al. $(2006,2008)$, in which we performed photometry with much higher photometric accuracy using deeper and higher-resolution $J H K$ images obtained with the Subaru $8.2 \mathrm{~m}$ telescope.

\subsection{Two Young Embedded Clusters}

Figure 2 shows the expanded images of both clusters. The Cloud 2-N cluster looks like a loose association similar to those in Taurus dark cloud (see, e.g., Lada, Strom, \& Myers 1993), while the Cloud 2-S cluster shows more

\footnotetext{
${ }^{6}$ IRAF is distributed by the National Optical Astronomy Observatories, which are operated by the Association of Universities for Research in Astronomy, Inc., under cooperative agreement with the National Science Foundation.

7 These two CO cores were originally called Cloud 2a and Cloud 2b, respectively, in Digel et al. (1994).
}

packed morphology like those in $\rho$ Oph star-forming regions (see, e.g., Allen et al. 2007), suggesting the Cloud $2-\mathrm{N}$ cluster is in isolated star formation mode, while Cloud 2-S cluster is in cluster star formation mode. The Cloud 2-N and Cloud 2-S clusters are distributed over a region of $\sim 1.5$ and $\sim 0.5 \mathrm{pc}$ in diameter with stellar surface densities of about 10 and $50 \mathrm{stars} \mathrm{pc}^{-2}$, respectively, assuming the heliocentric distance of $12 \mathrm{kpc}$. (See Yasui et al. 2008 for a more detailed analysis based on deeper images.) The density for Cloud 2-S is typical for young embedded clusters, but that of Cloud $2-\mathrm{N}$ is less than the lower boundary for clusters listed in Lada \& Lada (2003). There is a bright star at the center of the Cloud 2 -S cluster. Assuming a distance modulus of 15.4, the $K$-band luminosity of the brightest star is $M_{\mathrm{K}} \sim-0.4$, which is consistent with that of a late B-type star (Tokunaga 2000). Thus, the Cloud 2-S cluster seems to be a star cluster aggregating around a relatively massive star, most likely a Herbig Ae/Be star (see, e.g., Testi et al. 1999). If the heliocentric distance is $16.6 \mathrm{kpc}$ as derived from the recent H I data (Stil \& Irwin 2001), the above stellar densities are reduced by a factor of $\sim 2$, making the Cloud $2-\mathrm{N}$ cluster a very loose association $(\sim 5$ stars $\mathrm{pc}^{-2}$ ), while the Cloud 2-S cluster is still a dense cluster with a typical density $\left(\sim 25\right.$ stars $\left.\mathrm{pc}^{-2}\right)$. Also, the absolute magnitude of the brightest star of the Cloud 2-S cluster becomes $M_{\mathrm{K}} \sim-1.1$, which is consistent with that of an early to mid B-type star (Tokunaga 2000). Therefore, the above conclusion regarding isolated and cluster star formation modes still holds despite the possible distance uncertainty.

\subsection{Isolated Bright Young Stellar Objects}

Kobayashi \& Tokunaga (2000) found several bright young stellar objects (YSOs) in and around Cloud 2. Besides IRS 2 and 3, which are resolved as clusters in Cloud 2-S, other IRS sources (IRS 1, 4, and 5) are also detected and resolved in our new images (Fig. 3). Those bright isolated infrared stars are located eastward of the molecular cloud (see Fig. 1). Their $K$-band absolute magnitudes $\left(M_{\mathrm{K}}=-2.4,-1.3,-2.2\right.$; Kobayashi \& Tokunaga 2000) and $H-K$ excess suggest all of them are dust enshrouded intermediate-mass stars, probably Herbig $\mathrm{Ae} / \mathrm{Be}$ stars. IRS 1, which is just outside of Cloud $2-\mathrm{N}$, appears to be a single star, but it has an extended nebulosity in NE-SW direction (see the left panel in Fig. 3 ), suggesting it is a YSO with outflow activity. IRS 4 and 5, which are eastward of Cloud 2-S, have faint red companion stars to the west (see the middle and right panels in Fig. 3), suggesting they form typical small aggregations around intermediate-mass stars (Testi et al. 1999).

In our NIR images, IRS 1, 4, and 5, as well as MR-1, do not show a cluster with a considerable number (e.g., $>10$ ) of NIR sources at parsec scales as do the two embedded clusters. They appear to be isolated bright objects. Kobayashi \& Tokunaga (2000) argued that those bright YSOs are associated with Cloud 2 because of their proximity to the cloud and also because bright red sources were found only near the Cloud 2 in their entire surveyed area of $34^{\prime} \times 40^{\prime}$. Also one of the sources, IRS 5 , accompanies a small molecular cloud core extending from the Cloud 2-S core toward the southwest (Fig. 1). Therefore, their association with Cloud 2 appears to be 
quite firm.

\section{STAR FORMATION IN CLOUD 2}

\subsection{Overall Activity and Geometry}

Besides the clusters and the isolated YSOs, other star formation-related activities are known in the Cloud 2 region. Soon after the discovery of Cloud 2, de Geus et al. (1993) discovered an associated extended H II region with an $\mathrm{H} \alpha$ emission line, and a visible early-type star, MR-1, which had been already found by an independent blue star search in the outer Galaxy (Muzzio \& Rydgren 1974), was recognized as a probable exciting source of the H II region. Stil \& Irwin (2001) found extended $21 \mathrm{~cm}$ $(1.4 \mathrm{GHz})$ continuum emission projected on Cloud 2-N and that it is located on the maxima of the $\mathrm{H} \alpha$ intensity map of de Geus et al (1993). Based on the correlation with $\mathrm{H} \alpha$ and the absence of a counterpart at $74 \mathrm{~cm}$, Stil \& Irwin (2001) suggested that the continuum emission is thermal emission from an H II region.

Figure 4 summarizes the star formation activities in Cloud 2: the locations of NIR objects and MR-1 are overplotted on the H I $21 \mathrm{~cm}$ grayscale image, ${ }^{8} 1.4 \mathrm{GHz}$ radio continuum coutour, ${ }^{9}$ and ${ }^{12} \mathrm{CO}$ (1-0) contour from our Nobeyama data. Figure 4 clearly shows that the CO emission delineates the inner edge of the SNR H I shell, whose center is located toward the left bottom side of this image. Stil \& Irwin (2001) first suggested that Cloud 2 is associated with the approaching (blueshifted) side of the H I shell based on the coincidence of the sky position and the line-of-sight velocities $\left(v_{\mathrm{LSR}}=-103.6\right.$ $\mathrm{km} \mathrm{s}^{-1}$ for ${ }^{12} \mathrm{CO}$ and $=-101.9 \mathrm{~km} \mathrm{~s}^{-1}$ for $\left.\mathrm{H} \mathrm{I}\right)$. The $\mathrm{CO}$ cloud is located by the approaching side of the $\mathrm{H} \mathrm{I}$ shell as if it delineates the inner side of the shell (Fig. 4. see also Fig. 5). Because the radial velocities of $\mathrm{CO}$ and $\mathrm{H}$ I clouds are consistent with that for the $\mathrm{H}$ II region $\left(v_{\mathrm{LSR}}=-101 \mathrm{~km} \mathrm{~s}^{-1}\right.$ from Fabry-Perot $\mathrm{H} \alpha$ imaging; de Geus et al. 1993), it is highly likely that H I, CO, and the $\mathrm{H}$ II region are associated with each other at the same distance from us. The radial velocity of the visible earlytype star MR-1 was measured at $v_{\mathrm{LSR}}=-102.7 \pm 12 \mathrm{~km}$ $\mathrm{s}^{-1}$ by Russeil et al. (2007), and this high velocity is also quite consistent with the velocities of the warm and cold gas components. Some runaway OB stars with this velocity have been observed in the I Per OB association (McLachlan \& Nandy 1985), which is associated with the foreground Perseus arm but relatively close to Cloud 2 on the sky with an angular distance of about 3-5 degrees. However, because most of the OB stars in the association have $v_{\mathrm{LSR}}=-30$ to $-50 \mathrm{~km} \mathrm{~s}^{-1}$, which is the typical velocity for the Perseus arm, it would be quite safe to say that MR-1, with a much higher velocity, is associated with Cloud 2, which is located far beyond the Perseus arm. We can therefore conclude that all those objects in Figure 4 (from H I cloud to the NIR clusters) are associated each other at the same distance.

De Geus et al. (1993) found that the three intensity maxima of the $\mathrm{H} \alpha$ (in their Fig. 3), which are shown with squares in Figure 4, match well with the extended features of $1.4 \mathrm{GHz}$ continuum, which strongly support

8 From Canadian Galactic Plane Survey (CGPS) data at http://www1.cadc-ccda.hia-iha.nrc-cnrc.gc.ca/cgps/

9 From NRAO/VLA Sky Survey (NVSS) data at http://www.cv.nrao.edu/nvss/ (Condon et al. 1998). the suggestion by Stil \& Irwin (2001) that the radio continuum is coincident with the $\mathrm{H}$ II region in this starforming region. The radio continuum emissions extend from near the CO cloud to the east toward MR-1 (open star mark). This suggests that H II region is excited by MR-1 and partly by the IRS sources (filled green star marks). We have also noticed that one more strong radio continuum peak is located just 1-2 arcmin south of the Cloud 2-N cluster (Fig. 4). This strong peak is not seen in the $\mathrm{H} \alpha$ images by de Geus et al. (1993); rather, their image shows a local minimum of the $\mathrm{H} \alpha$ emission near this radio continuum peak. Therefore, the $\mathrm{H}$ II gas of this extended radio continuum feature may be located behind the $\mathrm{CO}$ cloud, which should hide the accompanying $\mathrm{H} \alpha$ emission by strong dust extinction. This view is consistent with the fact that all the stars in the Cloud 2-N cluster are behind the $\mathrm{CO}$ cloud with a uniform extinction of $A_{V} \sim 4$ mag as found by Yasui et al. (2008): ${ }^{10}$ we are seeing the Cloud 2-N cluster and the H II emission feature through the moderately thick molecular cloud. Unlike the Cloud 2-N, the Cloud 2-S does not seem to be under the strong influence of the photoionization by MR-1 and/or the bright NIR sources.

Figure 5 shows Cloud 2 and the cloud members on the IRAS $60 \mu \mathrm{m}$ image $^{11}$ as well as the H I emission contour of the SNR shell. An extended IRAS source, IRAS $02450+5816$, which is bright at 60 and $100 \mu \mathrm{m}$, is located between MR-1 and Cloud 2-N and was identified as a photodissociation region formed by MR-1 and/or IRS 1 (Kobayashi \& Tokunaga 2000). The Cloud 2-S cluster is in the vicinity of an unresolved IRAS source IRAS $02447+5811$, which is bright only in $60 \mu \mathrm{m}$ (see Table 2 in Kobayashi \& Tokunaga 2000) and is most likely related to the relatively hotter dust around the bright YSO in the center of the Cloud 2-S cluster. There might be some contribution to the IRAS flux of IRAS $02447+5811$ from other bright isolated YSOs, IRS 4 and 5, because its IRAS $60 \mu \mathrm{m}$ image appears to extend slightly toward those bright isolated YSOs (Fig. 5). We have checked mid-infrared images by the MSX (Midcourse Space Experiment $)^{12}$ at $8-21 \mu \mathrm{m}$, but could not find any clear counterpart to the Cloud 2-N and 2-S clusters as well as the bright isolated YSOs. Although the very extended $60 \mu \mathrm{m}$ features at the top of Figure 5 are accompanied mostly by unrelated foreground clouds and star-forming regions, some of the features (like the filamentary feature just outside the $\mathrm{H}$ I shell delineated by the dashed white line) might be related to the H I shell.

\subsection{Sequential Star Formation}

The CO cloud has an arc shape (Figs. 1, 4, and 5) that is well aligned with the H I SNR shell GSH 138-

10 They originally suggested a total extinction of $A_{V} \sim 7 \mathrm{mag}$ toward the Cloud 2-N cluster in Yasui et al. (2006), but the value became slightly smaller $\left(A_{V} \sim 6\right)$ after re-analyzing the data (Yasui et al. 2008). The contribution from Cloud 2 only is $A_{V} \sim 4$ mag after subtracting the contribution from the foreground ISM $\left(A_{V} \sim 2 \mathrm{mag}\right)$. This extinction is consistent with Ruffle et al. (2007), who suggested $A_{V}<4 \mathrm{mag}$ based on their submillimeter observations and a lower than average dust-to-gas ratio in the low-metallicity cloud.

11 From IRAS Galaxy Atlas at IPAC, JPL http://irsa.ipac.caltech.edu/data/IGA/

12 From MSX Data Atlas at IPAC, JPL http://irsa ipac.caltech.edu/data/MSX/1). 
01-94 and open toward the center of the shell (Fig. 5), showing that the CO cloud is clearly affected by the SNR $\mathrm{H}$ I shell. The clusters are associated with the two densest CO peaks of Cloud 2, and the sharp CO contours from the cluster toward the center of the SNR shell (Fig. 1; see also CO 2-1 map in Ruffle et al. 2007) suggest that the compression of molecular gas triggered the star formation. In Cloud 2-N, the cluster is associated with the northeastern subpeak, which appears to be more strongly compressed in the expansion direction than the southwestern subpeak, which does not harbor any clear star formation activity.

The young stars in and around Cloud 2 appear to show an age sequence from east to west following the direction of the SNR shell expansion. Compared to the visible early-type star MR-1, the bright NIR sources IRS 1, 4, and 5 apparently form a younger stellar population with redder colors and locations closer to the molecular cloud. Although IRS 5 may have a small molecular cloud core extending toward the southwest (see Figs. 1 and 44), all three sources are not directly associated with the massive molecular clouds as are the two embedded clusters. While the clusters are visible only in the near-infrared, we confirmed that IRS 1,4 , and 5 are marginally visible in the DSS2 $R$-band and IR-band plates. These facts suggest that the bright NIR sources are less embedded and older than the embedded clusters. In summary, there is an age sequence (old to young) from the visible early-type star, MR-1, to the isolated intermediate-mass infrared stars IRS 1, 4, and 5, and finally to the two embedded clusters in the molecular clouds. The direction of the age sequence following that of the SNR shell expansion (see Fig. (4) suggests a sequential star formation triggered by the supernova explosion.

Was the formation of MR-1 triggered by the SNR shell, or was it coincidentally situated there before the SN explosion? The age of the SNR shell was originally estimated at 4.3 Myr by Stil \& Irwin (2001). Because the expansion age is in proportion to the shell radius in Stil \& Irwin's model, ${ }^{13}$ it can be as low as $3.0 \mathrm{Myr}$ for our assumed distance $(D=12 \mathrm{kpc})$ or as much as $4.3 \mathrm{Myr}$ for the Stil \& Irwin's assumed distance $(D=16.6 \mathrm{kpc})$. On the other hand, it is difficult to estimate the age of MR-1. Its spectral type was estimated at B0-B1 (Muzzio \& Rydgren 1974; Smartt et al. 1996), suggesting that its mass is about $20 M_{\odot}$ and its age is no more than $10 \mathrm{Myr}$ if it is on the main-sequence. Smartt et al. (1996) suggested that judging from its low gravity, $\log g=3.7 \pm 0.1$, MR-1 is likely just evolving off the main sequence, while that of a B0-B1V star is $\log g=3.9$ (Drilling \& Landolt 2000). In this case, the age of MR-1 should be greater than $10 \mathrm{Myr}$, and the star should have been located there before the supernovae explosion. However, Russeil et al. (2007) recently re-estimated the spectral type of MR-1 as O9V (thus, higher effective temperature with about $2000 \mathrm{~K}$ increase) with newly obtained highresolution spectra. This suggests that MR-1 could well be still on the main-sequence. Because Smartt et al.'s suggestion was based on a 0.2 dex difference in gravity (with the uncertainty of $0.1 \mathrm{dex}$ ) and because there is the effective temperature uncertainty as shown by Russeil et al.'s new observation, it would be hard to conclude

${ }^{13}$ In their model the expansion radius $\mathrm{R}$ is in proportion to $t^{\frac{2}{7}}$ that MR-1 is just evolving off the main-sequence. In this case, the age of MR-1 could be much less than $10 \mathrm{Myr}$. Because there is no convincing method to measure the age of a star on the main-sequence, it would be difficult to precisely determine the age of MR-1 with the accuracy necessary to compare its age with that of the SNR shell. Although there is a small possibility that MR-1 formed in situ prior to the SN-explosion, we suggest that the formation of MR-1 was triggered by the SNR shell in view of the small probability that the sequence of young stellar objects observed is by chance. In this scenario, the age of MR-1 can be as much as the age of the SNR shell (3 Myr) and most likely about $2 \mathrm{Myr}$ in view of the location of MR-1 with respect to the current SNR shell (see discussion in 4.2 for the age estimate with the SNtrigger model). In $\S 4$ we are going to discuss the process of the SN-triggered star formation in Cloud 2 in more detail, assuming MR-1 is the first object formed by the SNR shell.

\section{SUPERNOVA TRIGGERED STAR FORMATION}

\subsection{Cloud Formation}

Stil \& Irwin (2001) concluded that the expanding H I shell, GSH 138-01-94, was formed by a supernova because of the nonexistence of any $\mathrm{OB}$ association inside the shell. Although there is no conclusive evidence for the supernova explosion itself, as there is for the nearby SNR H I shells with radio continuum and X-ray emission, this interpretation appears to be quite solid in view of the observed properties, e.g., the perfectly spherical shell with a very large diameter that can survive only in a very low gas density environment with little or no perturbation from other objects.

Stil \& Irwin (2001) suggested that Cloud 2 is associated with the approaching (blueshifted) side of the H I shell based on the coincidence of the sky position (see Fig. 5) and the line-of-sight velocities $\left(v_{\mathrm{LSR}}=-103.6 \mathrm{~km} \mathrm{~s}^{-1}\right.$ for ${ }^{12} \mathrm{CO}$ and $=-101.9 \mathrm{~km} \mathrm{~s}^{-1}$ for $\mathrm{H} \mathrm{I}$, while the center velocity of the expanding shell is $v_{\mathrm{LSR}}=-94.2 \mathrm{~km} \mathrm{~s}^{-1}$ ). Recently, Ruffle et al. (2007) concluded that the SNR H I shell has interacted with Cloud 2 based on extensively observed and modeled molecular chemistry for Cloud 2. They suggest that the chemistry of Cloud 2 is a direct result of shock fronts from the SNR H I shell propagating through the cloud sometime between $10^{3}-10^{4}$ years ago (see also Chapter 13 of Ruffle 2006). At the EOG, there is also no confusion with other objects because there is no complex star formation history and there is very low gas density and little perturbation from the spiral arms. Therefore, we can conclude that the H I shell and the molecular cloud are closely associated.

The shape of the molecular cloud follows the H I shell (Figs. 4 and 5), and this suggests that the formation of the molecular cloud itself was related to the SNR shell. Ruffle et al. (2007) considered the possibility that the cloud was formed by the H I shell from swept-up interstellar gas through Rayleigh-Taylor instabilities. However, it is expected that the formation of a molecular cloud is slowed down in the low-metallicity environment because the formation of $\mathrm{H}_{2}$ molecules requires dust particles (e.g., see $§ 4.6$ in Dyson \& Williams 1997), and there is less dust in the low-metallicity environment. Typically, it takes $30 \mathrm{Myr}$ to form a giant molecular cloud even under conditions of solar metallicity (e.g., Tielens 2005), 
Kobayashi et al.

and it could take even longer in Cloud 2 in view of its very low dust content (Ruffle et al. 2007). This formation timescale is much longer than the age of the SNR shell ( $\sim 3 \mathrm{Myr})$. Therefore, it is likely that the molecular cloud was already there before the SN explosion and that the SN shock that passed through the molecular cloud formed the shape of the cloud (P. Ruffle, private communication), although we cannot completely exclude the possibility that the molecular cloud was formed by the SN shock. The cloud formation process itself in such low-metallicity environment still remains an important open question.

\subsection{Star Formation}

The steep CO contour on the inner side of the SNR shell for both Cloud 2-N and 2-S (Figs. 1 and 4) strongly suggests that the compression of the molecular cloud was brought on by the H I shell expansion. Some portion of the compression could be produced by the stellar wind from the formation of the first star, MR-1, as a secondary process of the supernova explosion. The H II region (Fig. 44), which extends in and around Cloud 2-N, clearly shows that at least Cloud $2-\mathrm{N}$ is strongly affected by MR-1. However, Cloud 2-S does not have either an associated $\mathrm{H} \alpha /$ radio continuum (Fig. 4) or PDR (Fig. [5), which suggests that Cloud 2-S was compressed purely by the SNR shell.

If the passage of the SNR shell has triggered the cluster formation in Cloud 2, the upper-limit of the age of the cluster can be estimated from the projected angular difference of the cluster from the current SNR shell front. Figure 6] shows that the projected angular difference is about 300 arcsec. Because the radius of the H I SNR shell $(2238$ arcsec) and its age is estimated at $3.0 \mathrm{Myr}$ for our assumed distance $D=12 \mathrm{kpc}$ (see discussion in (3.2), the projected angular difference suggests that the cluster is younger than $300 / 2238 \times 3.0 \sim 0.4 \mathrm{Myr}$. Although the shell expansion speed is expected to decline with time, that only shortens the upper-limit. Therefore, the age upper-limit of $0.4 \mathrm{Myr}$ still holds. It is very interesting to note that this age estimate is in quite good agreement with an independent age estimate of Cloud 2$\mathrm{N}$ cluster by Yasui et al. (2006), who estimated the age of the cluster at about $0.5 \mathrm{Myr}$ from the modeling of the $K$-band luminosity function. This consistency strongly supports the idea that the cluster formation was basically triggered by the SNR. Note that the SN shell would have moved more slowly through the denser material of Cloud 2 because of mass conservation. This possible slow expansion may cause some systematic uncertainty of the above age estimate.

\subsection{Isolated and Cluster Mode Star Formation}

The two embedded clusters show a distinct morphology difference: The one in the northern molecular cloud core is a loose association with an isolated star formation mode, while the other in the southern molecular cloud core is a dense cluster with cluster star formation mode. Because the cloud mass of both Cloud 2-N and 2-S are similar $\left(\sim 5 \times 10^{3} M_{\odot} ;{ }^{14}\right.$ Digel et al. 1994; Ruffle et al. 2007), the difference in the appearance of the two clusters

\footnotetext{
14 Originally Digel et al. (1994) estimated the cloud mass $10^{4} M_{\odot}$ assuming the kinematic distance of $D=21 \mathrm{kpc}\left(R_{\mathrm{g}}=\right.$
}

may give us a good opprotunity to study what causes the differences between the two star formation modes.

In Figure 7 we note that Cloud 2-N is distributed along the tangential direction of the SNR shell, while Cloud 2$\mathrm{S}$ extends slightly inward. A close investigation of the SNR H I shell in a velocity range similar to that of the CO clouds ( -102 to $\left.-105 \mathrm{~km} \mathrm{~s}^{-1}\right)$, revealed that another shell-like structure is associated with Cloud 2-S (Fig. 8). This substructure in the H I map can be also seen in the $\mathrm{H}$ I velocity channel map at $-104.5 \mathrm{~km} \mathrm{~s}^{-1}$ in Figure 1 in Stil \& Irwin (2001). We denote it as Cavity 2B since it encompasses Cavity 2 in the Stil \& Irwin paper. A closer look at the molecular cloud (Fig. 7) shows that the southern half of Cloud 2 appears to be perturbed by Cavity $2 \mathrm{~B}$ at around $\left(\mathrm{RA}_{\mathrm{J} 2000.0}\right.$, Dec $\left._{\mathrm{J} 2000.0}\right)=\left(2^{\mathrm{h}} 48^{\mathrm{m}} 30^{\mathrm{s}}\right.$, $\left.58^{\circ} 26^{\prime}\right)$.

We also found that both embedded clusters in Cloud 2 (Cloud 2-S cluster and the IRS 3 mini-cluster) are located on the western side of the cloud cores (see the distribution of red crosses in Fig. 7). This appears to imply that the shock from the western side, presumably from Cavity $2 \mathrm{~B}$, caused the star formation in Cloud 2-S. Therefore, we propose that strong compressions of Cloud 2-S from the combination of the SNR shell and Cavity $2 \mathrm{~B}$ caused the cluster-mode star formation whereas an isolated-mode star formation occurred in Cloud 2-N. This may be one of the best examples that clearly supports the theoretical and observational suggestions that high-pressure is the trigger of cluster-mode star formation (e.g., Elmegreen 1998, 2004).

The center of Cavity $2 \mathrm{~B}$ is at about $(\mathrm{l}, \mathrm{b})=$ $(137.59,-1.16)$ or $\left(\mathrm{RA}_{\mathrm{J} 2000.0}, \operatorname{Dec}_{\mathrm{J} 2000.0}\right)=\left(02^{\mathrm{h}} 46^{\mathrm{m}} 53^{\mathrm{s}}\right.$, $+58^{\circ} 23^{\prime}$ ) with a radius of about $12^{\prime}$ (about $45 \mathrm{pc}$ ). Cavity $2 \mathrm{~B}$ could be either a smaller (thus, younger) adjacent SNR shell or a hole on the SNR shell like Cavity 1 and 2 in Stil \& Irwin (2001). Although Cavity 1 appears to be a hole on the SNR shell rather than another SNR shell (Stil \& Irwin 2001), we confirmed a sign of an expanding shell for Cavity 2B in an H I position-velocity map made with the CGPS data. Because we could not identify any radio continuum source inside Cavity $2 \mathrm{~B}$ using the NVSS map (VLA 1.4 GHz), Cavity 2B does not seem to be powered by star-forming regions, and it could be another young SNR shell. Because this area around $(1, b)$ $=(137.5,-1.0)$ on the SNR shell appears to be crowded with H I clouds throughout the velocity range of the expanding shell (see Fig. 1 in Stil \& Irwin 2001), another interpretation is that Cavity $2 \mathrm{~B}$ is just a group of in situ $\mathrm{H}$ I clouds that were distributed before the SN explosion. Cloud 2 powered by the main SNR shell (GSH 138-0194) may have collided with the H I clouds, which caused the strong compression that stimulated the formation of the Cloud 2-S clusters.

\subsection{Summary of the SNR-triggered Star Formation History}

Figure 9summarizes our interpretation of the star formation history in Cloud 2. The first-generation star

$28 \mathrm{kpc})$. The presented number was converted from the original estimate assuming the distance of $D=12 \mathrm{kpc}\left(R_{\mathrm{g}}=19 \mathrm{kpc}\right)$. Ruffle et al. (2007) also calculate the cloud mass of both Cloud $2-\mathrm{N}$ and $2-\mathrm{S}$ as $\sim 5 \times 10^{3} \mathrm{M}_{\odot}$ for the distance of 14 to $20 \mathrm{kpc}\left(R_{\mathrm{g}}=\right.$ 22 to $28 \mathrm{kpc}$ ) using observed column densities and LVG models. 
(MR-1) and second-generation stars (the bright NIR sources) do not seem to accompany a cluster like that for the third-generation stars in Cloud 2-N and 2-S clusters. This may suggest that those early generation stars were born in an isolated mode without accompanying clusters. Although the ages of the clusters were estimated at $\sim 0.5$ Myr (see discussion in $\$ 4.2$ ), it is hard to determine when those early generation high- to intermediate-mass stars were born. In view of their possible locations inside the SNR sphere (the projected distance from the shell center to the stars is about $75 \%$ of the shell radius), their ages should not be more than 2 Myr in view of the age of the SNR shell ( $\sim 3$ Myr; see $\S 4.2)$ even after considering the slowing down of the shell expansion. This probable age for the second generation bright NIR sources is consistent with our interpretation that they are Herbig Ae/Be stars, whose typical ages are 0.1 to a few Myrs (van den Ancker et al. 1997). The probable age of $<2$ Myr is also consistent with the first generation star, MR1 , which is most likely in the main-sequence phase in view of its very short pre-main-sequence timescale $(\sim 0.1$ Myr; Bernasconi \& Maeder 1996) for the large mass $(\sim 20$ $\left.M_{\odot}\right)$. Most of the red sources scattered in the field in Figure 1 are background galaxies, although some of them might be stars formed with the first and second generation high/intermediate-mass stars. Note that the faint companions of the bright NIR stars, IRS 4 and 5, are distributed to the west. This might show the trace of the progressive star formation: first bright intermediatemass stars were born, then the low-mass stars just to the west formed from the remaining cloud. Future kinematical study of all those cloud members with NIR echelle spectroscopy may shed light on the dynamical processes that happened during the triggered star formation.

\section{LINK TO THE EARLY PHASE OF THE GALAXY'S FORMATION}

Ferguson, Gallagher, \& Wyse (1998) have pointed out that the outskirts of spiral galaxies in the local universe have the characteristics similar to high-redshift damped Lyman- $\alpha$ systems as well as to giant low surface brightness galaxies in that they have low gas surface densities (yet high gas fractions compared to stars), low metallicities, and long dynamical timescales. The irregular (Im) dwarf galaxies, which are generally dominated in the optical by their younger stellar populations, also have the similar characteristics (Hunter et al. 2006). They are usually lower in luminosity and surface brightness, more gas-rich with a lower metallicity and dust content, and form stars without the benefit of spiral density waves. All these characteristics suggest that the irregular dwarf galaxies are representative of the nature of star formation in the early universe (Hunter et al. 2006; Hunter \& Elmegreen 2004, 2006). However, the distance to the nearest Im galaxy (LMC) is $50 \mathrm{kpc}$, while the distance to the EOG is $10-20 \mathrm{kpc}$. The proximity of the EOG enables us to resolve star cluster members with groundbased seeing resolution (see Yasui et al. 2006, 2008) and spectroscopic study is also possible with the 8-meter class telescopes even for low-mass stars (e.g., down to $K=19$ to $20 \mathrm{mag}$ ). The gas content can be also studied in detail statistically (Snell et al. 2002) and with various molecular lines (Lubowich et al. 2004; Ruffle et al. 2005; Brand \& Wouterloot 2007). Therefore, the EOG is an excellent place to study the star formation processes that were present during the early epoch of galaxy formation and that are still present in Im galaxies (Hunter et al. 2006).

The metallicity of outer galaxy disks, Im galaxies, lowmetallicity blue compact dwarf galaxies (Kunth \& Ostlin 2000 ), and damped Lyman- $\alpha$ systems are observed down to $[\mathrm{Fe} / \mathrm{H}] \sim-3$ (e.g., Pettini 2004). This metallicity range traces the critical phases of early Galaxy formation when the major components, such as halo, thick disk, bulge, and thin disk, formed (e.g., Fig. 3 in Buser 2000; Freeman \& Bland-Hawthorn 2002). The typical metallicity of the outer galaxy regions $(-1.5<[\mathrm{Fe} / \mathrm{H}]$ $<-0.5)$ suggest that they represent the late phase of halo formation and the early phase of thick disk formation. Despite many extensive studies (e.g., Bensby et al. 2007, and references therein), the origin and role of the thick disk in our Galaxy are not well understood even decades after its discovery (Gilmore \& Reid 1983). Although the study of abundance patterns recorded in very low metallicity stars $([\mathrm{Fe} / \mathrm{H}]<-2.5)$ provides a vital clue to the star formation process in the very early epoch of galaxy formation (Audouze \& Silk 1995; Shigeyama \& Tsujimoto 1998; Tsujimoto \& Shigeyama 1998), this successful "archeological method" cannot be applied to the metallicity range of the thick disk because the abundance patterns of the formed stars are significantly affected by the Galactic global chemical evolution of the interstellar medium, from which the stars are formed. The observational study of the outer Galaxy region may directly reveal the details of the star formation process during the formation of the thick disk, which took place at $z \sim 2$ $(\sim 10$ Gyr ago), to shed light on the origin and role of this important Galactic component.

In the last decade, the formation of Population III stars (the "first stars") has been extensively studied theoretically (Abel, Brian \& Norman 2002; Bromm, Coppi, \& Larson 2002; Nakamura \& Umemura 2001) not only for its own sake, but because of its direct relation to the reionization of the early Universe and galaxy formation at an early epoch (see, e.g., Yoshida 2006). The recent advancement in the study of the extreme metalpoor stars (Christlieb et al. 2002; Beers \& Christlieb 2005), which are the potential relics of the Pop III stars, boosted the examination of early-phase galaxy formation through the study of abundance patterns recorded in very low metallicity stars at a very early epoch. Although theoretical studies suggest that the star formation physics in the metallicity range of the outer galaxy regions should not significantly differ from that for the solar metallicity (e.g., Omukai 2000), the observational study of the regions in this "niche" metallicity range establishes a good link between very low and solar metallicity ranges, thereby revealing important astronomical processes in this metallicity range.

It is most likely that SN-triggered star formation was the major process by which stars formed in the early epoch of galaxy formation because of the lack of other major star formation triggers such as density waves in spiral galaxies. Tsujimoto, Shigeyama \& Yoshii (1999) first formulated the SN-triggered star formation in the early epoch of Galaxy formation by utilizing the abundance pattern recorded in the very low metallicity stars in our Galaxy (see also Tsujimoto, Shigeyama \& Yoshii 2002). They successfully constrained the star formation 
Kobayashi et al.

efficiency and the mass function during the formation of the Galactic halo. With the clear SN-triggered star formation signatures, Cloud 2 and probably other EOG clouds are excellent places to confirm the predictions from near-field cosmology.

\section{CONCLUSION}

We present a detailed study of star formation in Cloud 2 , which is one of the active star-forming regions in the EOG and is one of the farthest star-forming regions, with a probable Galactic radius of $\sim 20 \mathrm{kpc}$. Cloud 2 has a quite different environment from that of the solar neighborhood, including lower metallicity, much lower gas density, and small or no perturbation from spiral arms. As such, it is a useful analog for the star formation process in an early epoch of our Galaxy. In particular, the study of the EOG may shed light on the origin and role of the thick disk, whose metallicity range matches well with that of the EOG.

Our main results are

1. With new wide-field near-infrared (NIR) imaging that covers the entire Cloud 2, we discovered two young embedded star clusters located in the two dense cores of the cloud.

2. The two embedded clusters show a distinct morphology difference: The one in the northern molecular cloud core is a loose association with an isolated star formation mode, while the other in the southern molecular cloud core is a dense cluster with a cluster star formation mode.
3. Using our NIR and ${ }^{12} \mathrm{CO}$ data as well as $\mathrm{H} \mathrm{I}$, radio continuum, and IRAS data from the archives, we show clear evidence of sequential star formation triggered by the large nearby supernova remnant, GSH 138-01-94.

4. We propose that the high compression resulting from a combination of the SNR shell and an adjacent shell caused the dense cluster formation in the southern core.

We are grateful to UH $2.2 \mathrm{~m}$ telescope staff, especially John Dvorak and Dr. Andrew Pickles for their support during the QUIRC observations. We also thank Takuji Tsujimoto for the fruitful discussions and suggestions about star formation in the early epoch of the Galaxy's formation. We thank the anonymous referee for careful reading and thoughtful suggestions that significantly improved this paper. This research was supported by a Grant-in-Aid for Encouragement of Scientists (A) of the Ministry of Education, Science, Culture, and Sports in Japan (No.11740128). NK was partly supported by a JSPS (Japan Society for the Promotion of Science) postdoctoral fellowship for research abroad. NK is also very grateful to the staff of the Institute for Astronomy, University of Hawaii for their warm support during his stay in Honolulu.

\section{REFERENCES}

Abel, T., Bryan, G. L., \& Norman, M. L. 2002, Science, 295, 93

Allen, L., et al. 2007, in Protostars and Planets V, ed. B. Reipurth, D. Jewitt \& K. Keil (Tucson: Univ. Arizona Press), 361

Audouze, J., \& Silk, J. 1995, ApJ, 451, L49

Beers, T. C., \& Christlieb, N. 2005, ARA\&A, 43, 531

Bensby, T., Zenn, A. R., Oey, M. S., \& Feltzing, S. 2007, ApJ, 663, L13

Bernasconi, P. A., \& Maeder, A. 1996, A\&A, 307, 829

Brand, J., \& Wouterloot, J. G. A. 1995, A\&A, 303, 851

Brand, J., \& Wouterloot, J. G. A. 2007, A\&A, 464, 909

Brewer, M.-M., \& Carney, B. W. 2006, AJ, 131, 431

Bromm, V., Coppi, P. S., \& Larson, R. B. 2002, ApJ, 564, 23

Buser, R. 2000, Science, 287, 69

Christlieb, N., et al. 2002, Nature, 419, 904

Condon, J. J., Cotton, W. D., Greisen, E. W., Yin, Q. F., Perley R. A., Taylor, G. B., \& Broderick, J. J. 1998, AJ, 115, 1693

de Geus, E. J., Vogel, S. N., Digel, S. W., \& Gruendl, R. A. 1993, ApJ, 413, L97

Digel, S., de Geus, E., \& Thaddeus, P. 1994, ApJ, 422, 92

Drilling, J. S., \& Landolt, A. U. 2000, in Allen's Astrophysical Quantities, ed. A. N. Cox (4th ed., New York: AIP Press)

Dyson, J. E., \& Williams, D. A. 1997, The Physics of the Interstellar Medium (2nd ed., Bristol: Institute of Physics)

Elmegreen, B. C. 2004, in ASP Conf. Ser. 322, The Formation and Evolution of Massive Young Star Clusters, eg. H. J. G. L. M. Lamers, L. J. Smith, \& A. Nota (San Francisco: ASP), 277

Elmegreen, B. G. 2002, ApJ, 577, 206

Elmegreen, B. G. 1998, in ASP Conf. Ser. 148, Origins, ed. C. E. Woodward, J. M. Schull, \& H. A. Thronson, Jr. (San Francisco: ASP), 150

Ferguson, A. M. N., Gallagher, J. S., \& Wyse, R. F. G. 1998, AJ, 116,673

Freeman, K., \& Bland-Hawthorn, J. 2002, ARA\&A, 40, 487

Gilmore, G., \& Reid, N. 1983, MNRAS, 202, 1025

Heyer, M. H., Brunt, C., Snell, R. L., Howe, J. E., \& Schloerb, F. P 1998, ApJS, 115, 241

Hunter, D. A., \& Elmegreen, B. G. 2006, ApJS, 162, 49
Hunter, D. A., \& Elmegreen, B. G. 2004, AJ, 128, 2170

Hunter, D. A., Elmegreen, B. G., \& Martin, E. 2006, AJ, 132, 801

Kobayashi, N., \& Tokunaga, A. T. 2000, ApJ, 532, 423

Kobayashi, N., \& Tokunaga, A. T. 2001, in ASP Conf. Ser. 231, Tetons 4: Galactic Structure, Stars and the Interstellar Medium, ed. C. E. Woodward, M. D. Bicay, \& J. M. Schull (San Francisco: ASP), 83

Kunth, D., \& Ostlin, G. 2000, A\&A Rev., 10, 1

Lada, C. J., \& Lada, E. A. 2003, ARA\&A, 41, 57

Lada, E. A., Strom, K. M., \& Myers, P. C. 1993, in Protostars and Planets III, ed. E. H. Levy, \& J. I. Irving (Tucson: Univ. Arizona Press), 245

Lubowich, D. A., Brammer, G., Roberts, H., Millar, T. J., Henkel, C., \& Pasachoff, J. M. 2004, in Origin and Evolution of the Elements, ed. A. McWilliam, \& M. Rauch (Pasadena: Carnegie Observatories), 37 http://www.ociw.edu/ociw/symposia/series/symposium4/proceedings.html McLachlan, A., \& Nandy, K. 1985, MNRAS, 215, 473

Muzzio, J. C., \& Rydgren, A. E. 1974, AJ, 79, 864

Nakamura, F., \& Umemura, M. 2001, ApJ, 548, 19

Nakanishi, H., \& Sofue, Y. 2003, PASJ, 55, 191

Omukai, K. 2000, ApJ, 534, 809

Pettini, M. 2004, in Cosmochemistry: The Melting Pot of the Elements, ed. C. Esteban, R. J. Garcí López, A. Herrero, F. Sánchez (Cambridge, UK: Cambridge University Press), 257

Rolleston, W. R. J., Smartt, S. J., Dufton, P. L., \& Ryans, R. S. I. 2000, A\&A, 363, 537

Ruffle, P. 2006, Ph.D. thesis, Univ. Manchester.

Ruffle, P., Millar, T., Roberts, H., Henkel, C., \& Lubowich, D. 2005, IAU Symposium, 231, 1

Ruffle, P. M. E., Millar, T. J., Roberts, H., Lubowich, D. A., Henkel, C., Pasachoff, J. M., \& Brammer, G. 2007, ApJ, 671, 1766

Russeil, D., Adami, C., \& Georgelin, Y. M. 2007, A\&A, 470, 161

Ryan, S. G., Norris, J. E., \& Beers, T. C. 1996, ApJ, 471, 254

Santos, C. A., Yun, J. L., Clemens, D. P., \& Agostinho, R. J. 2000, ApJ, 540, L87 
Shigeyama, T., \& Tsujimoto, T. 1998, ApJ, 507, L135

Smartt, S. J., Dufton, P. L., \& Rolleston, W. R. J. 1996, A\&A, 305, 164

Smartt, S. J., \& Rolleston, W. R. J. 1997, ApJ, 481, L47

Snell, R. L., Carpenter, J. M., \& Heyer, M. H. 2002, ApJ, 578, 229

Stil, J. M. \& Irwin, J. A. 2001, ApJ, 563, 816

Testi, L., Palla, F., \& Natta, A. 1999, A\&A, 342, 515

Tielens, A. G. G. M. 2005, The Physics and Chemistry of the Interstellar Medium (Cambridge, UK: Cambridge University Press)

Tokunaga, A. T. 2000, in Allen's Astrophysical Quantities, ed. A. N. Cox (4th ed., New York: AIP Press)

Tsujimoto, T., \& Shigeyama, T. 1998, ApJ, 508, L151

Tsujimoto, T., Shigeyama, T., \& Yoshii, Y. 1999, ApJ, 519, L63

Tsujimoto, T., Shigeyama, T., \& Yoshii, Y. 2002, ApJ, 565, 1011 van den Ancker, M. E., The, P. S., Tjin A Djie, H. R. E., Catala, C., de Winter, D., Blondel, P. F. C., \& Waters, L. B. F. M. 1997, A\&A, 324, L33

Westerhout, G., \& Wendlandt, H.-U. 1982, A\&AS, 49, 143

Wouterloot, J. G. A., \& Brand, J. 1989, A\&AS, 80, 149

Wouterloot, J. G. A., Brand, J., Burton, W. B., \& Kwee, K. K. 1990, A\&A, 230, 21

Yasui, C., Kobayashi, N., Tokunaga, A. T., Terada, H., \& Saito, M. 2006, ApJ, 649, 753

Yasui, C., Kobayashi, N., Tokunaga, A. T., Terada, H., \& Saito, M. 2008, ApJ, 675, 443

Yoshida, N. 2006, New Astron. Rev., 50, 19 


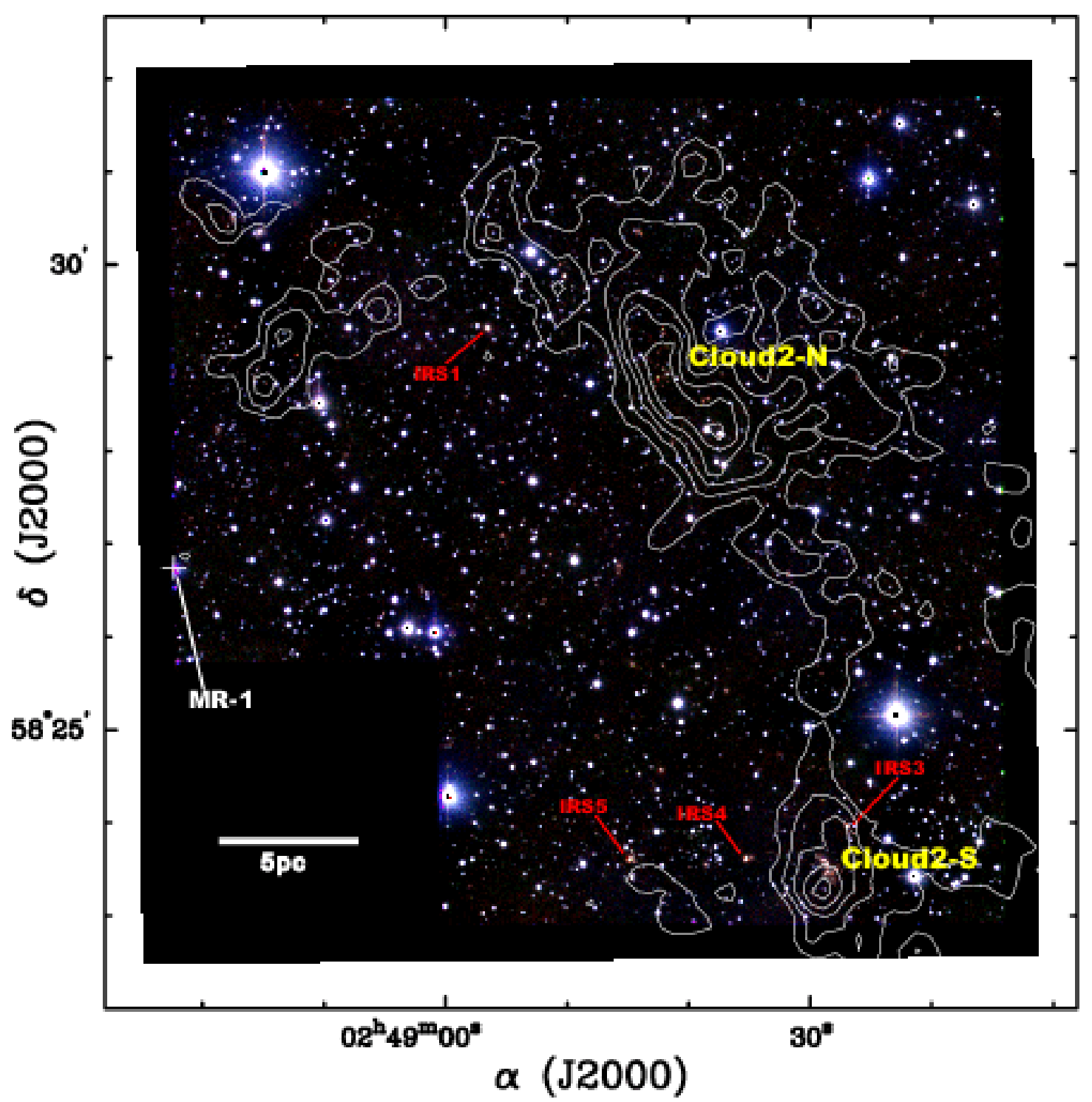

FIG. 1. - Near-infrared $J H K$ three-color image of Cloud 2 obtained with QUIRC near-infrared camera. North is up, and east is to the left. ${ }^{12} \mathrm{CO}$ (1-0) data obtained with Nobeyama $45 \mathrm{~m}$ telescope is overplotted as the white contour. The locations of bright NIR sources (IRS 1, 4, 5; Kobayashi \& Tokunaga 2000) and a visible early-type star MR-1 are also indicated. A loosely packed embedded cluster (Cloud 2-N cluster) is seen as an aggregation of red sources in the northern $\mathrm{CO}$ peak of the two CO peaks in the Cloud 2-N molecular cloud. A dense embedded cluster (Cloud 2-S cluster) is seen near the CO peak of the Cloud 2-S molecular cloud. This cluster was originally identified as IRS 2 in Kobayashi \& Tokunaga (2000). IRS 3 near Cloud 2-S was also found to be a very small cluster (see also Fig. 2). Most of other faint red sources in this field are faint background galaxies. The NIR image was Gaussian-convolved for viewing purpose. The resultant FWHM of the image is about $0{ }^{\prime \prime} 7$. 

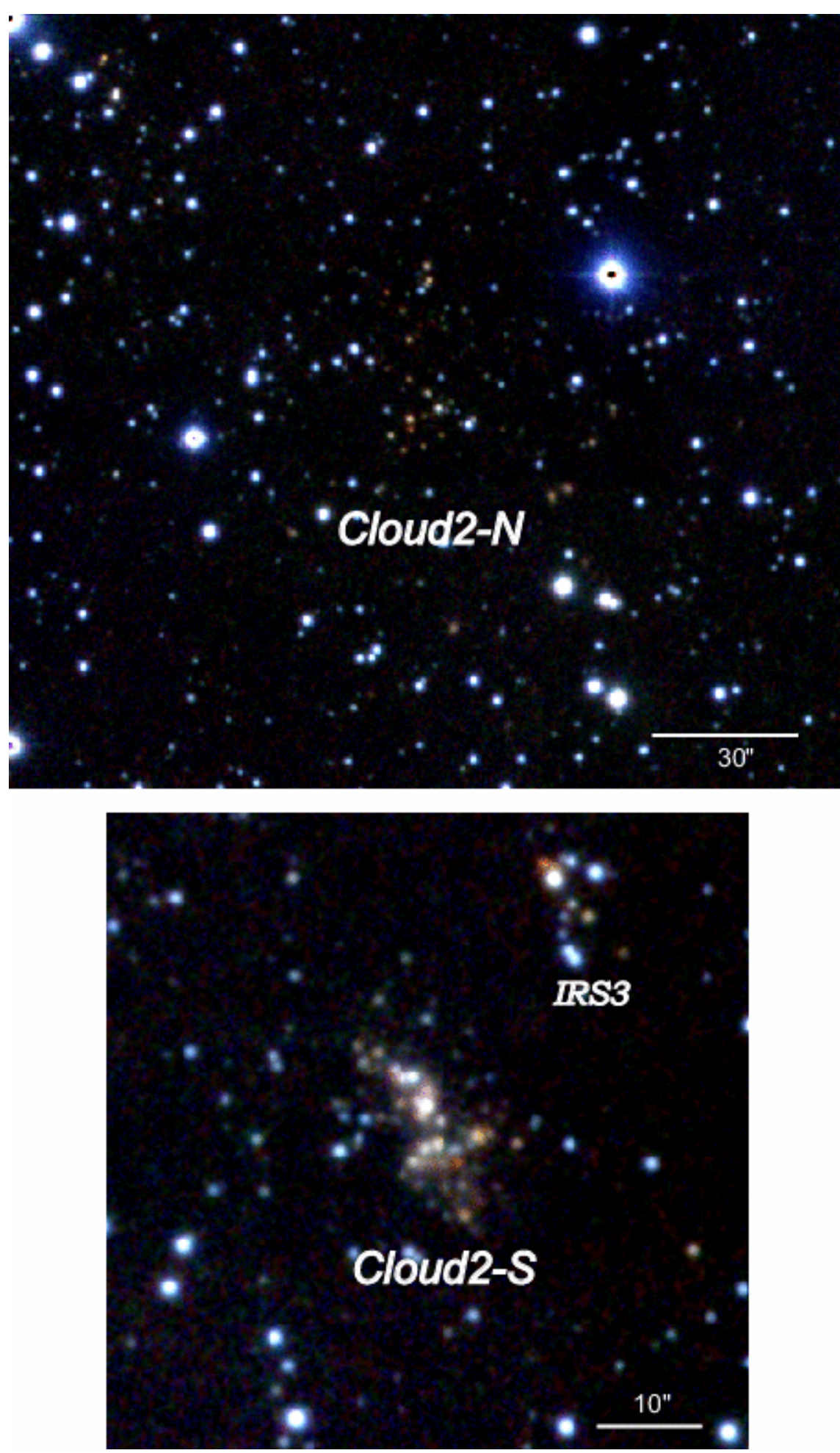

FIG. 2.- The expanded view of the JHK three-color image of the two embedded clusters (Left: Cloud 2-N cluster, Right: Cloud 2-S cluster). Note the difference of stellar density in those two clusters. IRS 3 near the Cloud 2-S cluster is resolved into a very small cluster in this image. 
Kobayashi et al.
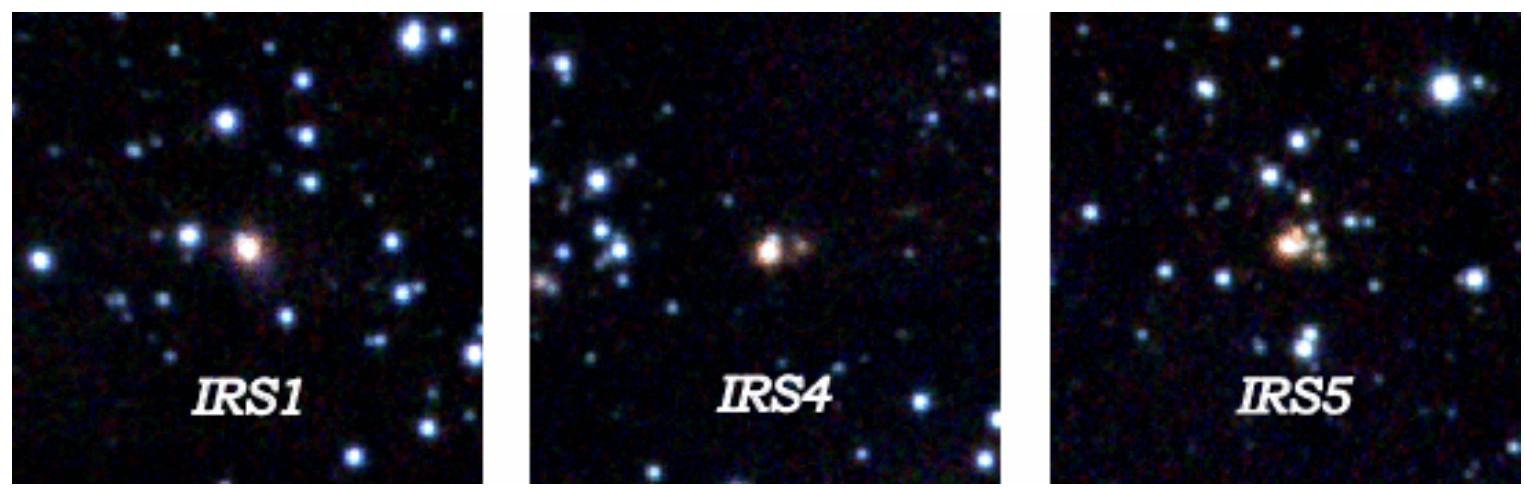

FIG. 3. - The expanded view of the $J H K$ three-color image of the bright NIR sources near Cloud 2. Each box shows $30^{\prime \prime}$ square. North is up, and east is to the left. These sources are identified as intermediate-mass YSOs (Herbig Ae/Be stars). IRS 1 shows a faint nebulosity extended to southwest, which would be an outflow or a reflection nebula. IRS 4 and 5 show several faint red companion stars to the west, forming very small clusters. 


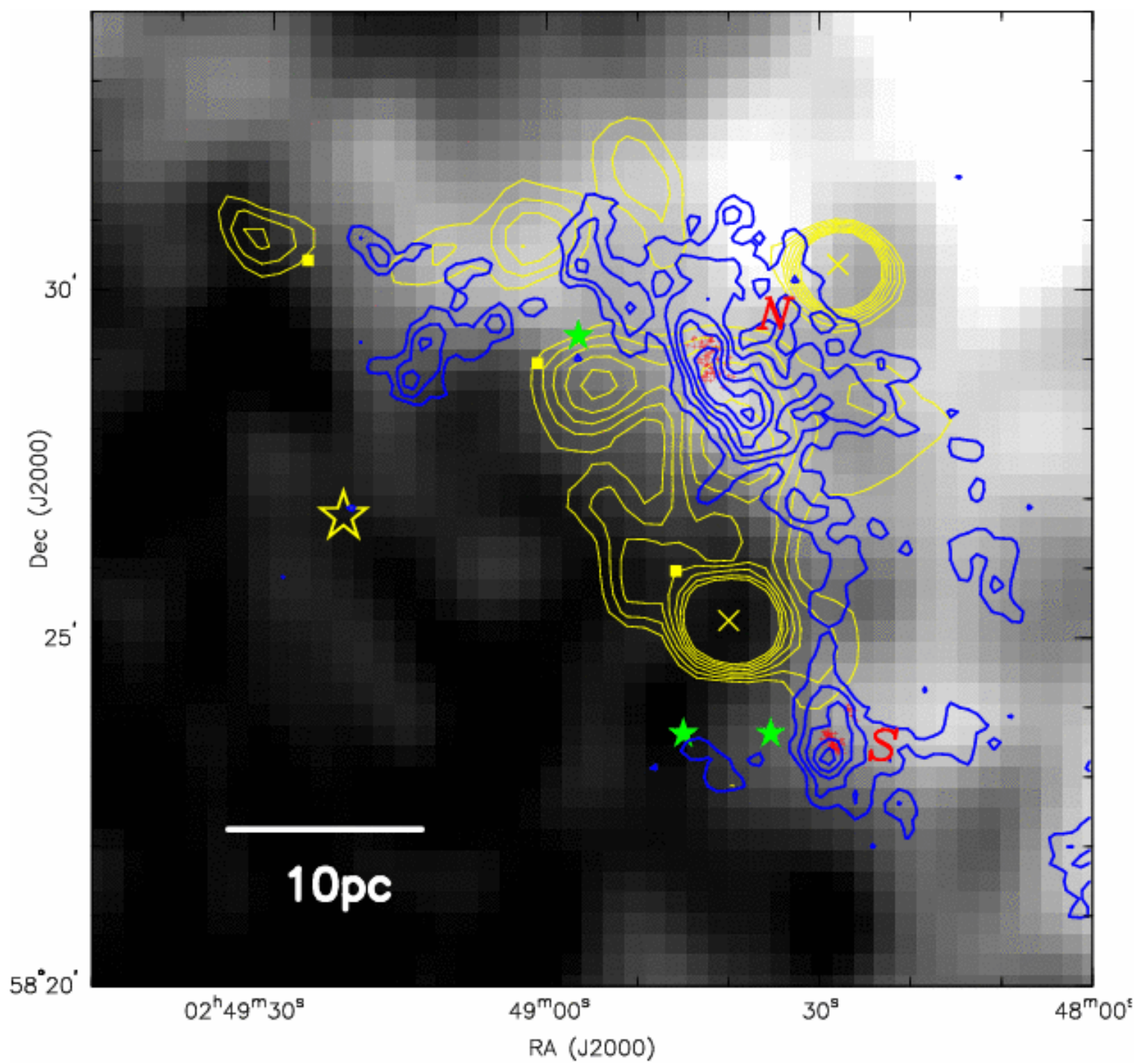

FIG. 4.- Summary of star formation activities in Cloud 2. A grayscale image and blue contours show the distribution of $\mathrm{H}$ I and ${ }^{12} \mathrm{CO}$ (1-0) emission integrated over the velocity range -94 to $-105 \mathrm{~km} \mathrm{~s}^{-1}$. Yellow contours show $1.4 \mathrm{GHz}$ radio continuum from NVSS (NRAO VLA Sky Survey) data: Note that the two brightest pointlike sources with cross marks are unrelated foreground/background radio sources. Filled yellow squares show the peaks of $\mathrm{H} \alpha$ nebula (de Geus et al. 1993). The open yellow star and filled green stars show MR-1 and bright NIR sources, respectively. The locations of the members in the Cloud 2-N, Cloud 2-S clusters, and the Cloud 2-S mini-cluster are shown with red crosses. [Refer to the ApJ on-line PDF to see the detail of the distribution of the cluster members] Although the spatial-resolution of the H I image and the VLA map are originally $1^{\prime}$ and $45^{\prime \prime}$, respectively, here they are Gaussian-convolved with 1-pixel (18" and $15^{\prime \prime}$, respectively) for better viewing. The beamsizes of the Nobeyama CO map is about $20^{\prime \prime}$. See the discussion in the main text for detail. 


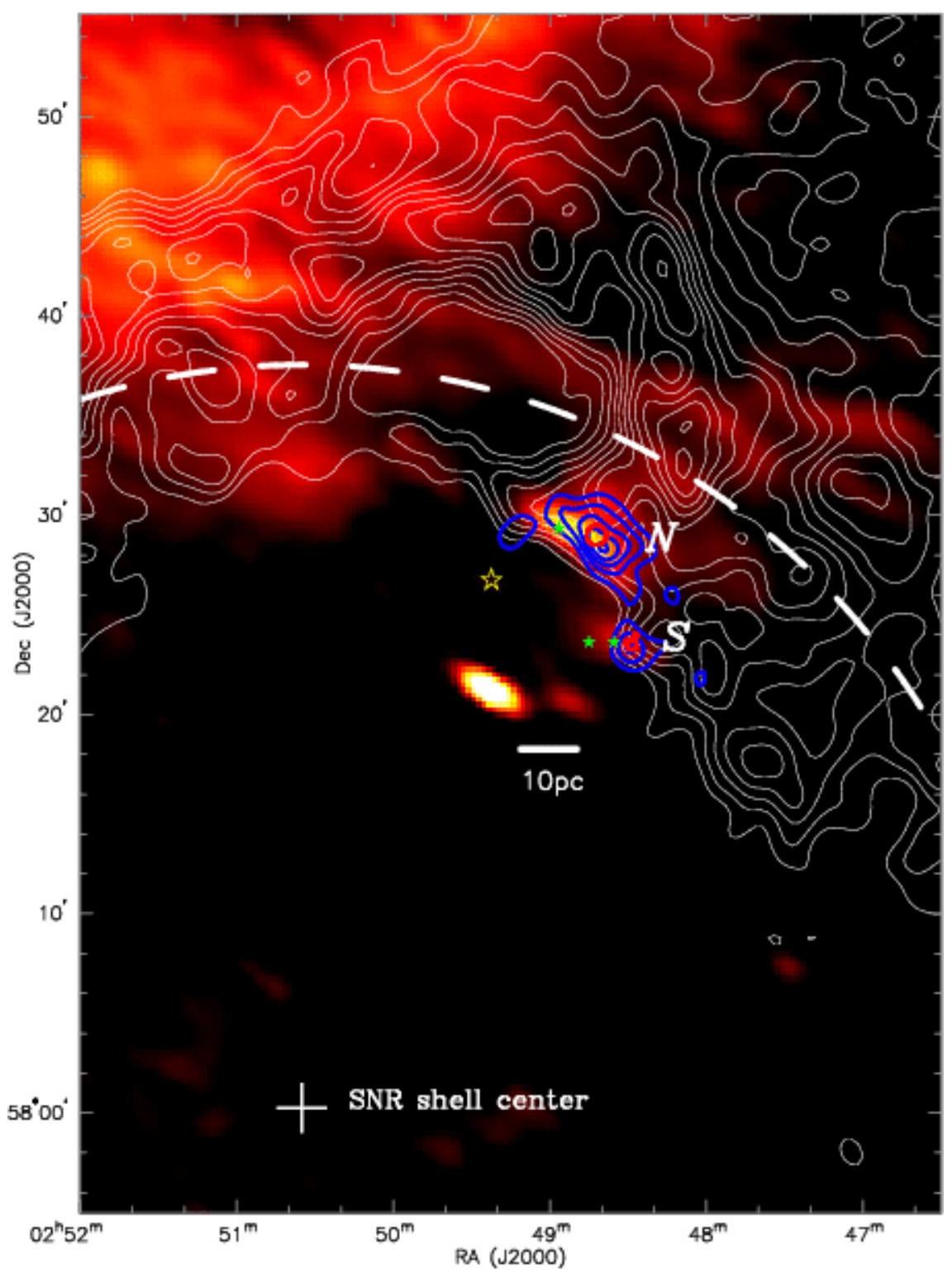

Fig. 5.- The locations of Cloud 2 and forming stars with respect to the SNR shell GSH 138-01-94 (Stil \& Irwin 2001). The center of the shell is shown with a white cross, while the edge of the shell is shown with a dashed line. White contours show the H I surface brightness integrated over the velocity range $v_{\mathrm{LSR}}=-94$ to $-105 \mathrm{~km} \mathrm{~s}^{-1}$ (from CGPS data), while the blue contours show the ${ }^{12} \mathrm{CO}(1-0)$ surface brightness (from our Nobeyama data). The background image is the IRAS $60 \mu \mathrm{m}$ image from IRAS IGA data. The open yellow star mark, filled green star marks, and red circles show the locations of the visible early-type star MR-1, the bright NIR sources, and the two embedded clusters, respectively. The H I and IRAS images are 1- and 2-pixel Gaussian-convolved, respectively, for better comparison to the CO map. The bright IRAS source in the center of the image is an unrelated foreground object (a combination of two bright NIR sources, IRS 6 and 7 in Kobayashi \& Tokunaga 2000), and shows the average PSF of this IRAS image. See the discussion in the main text for detail. 


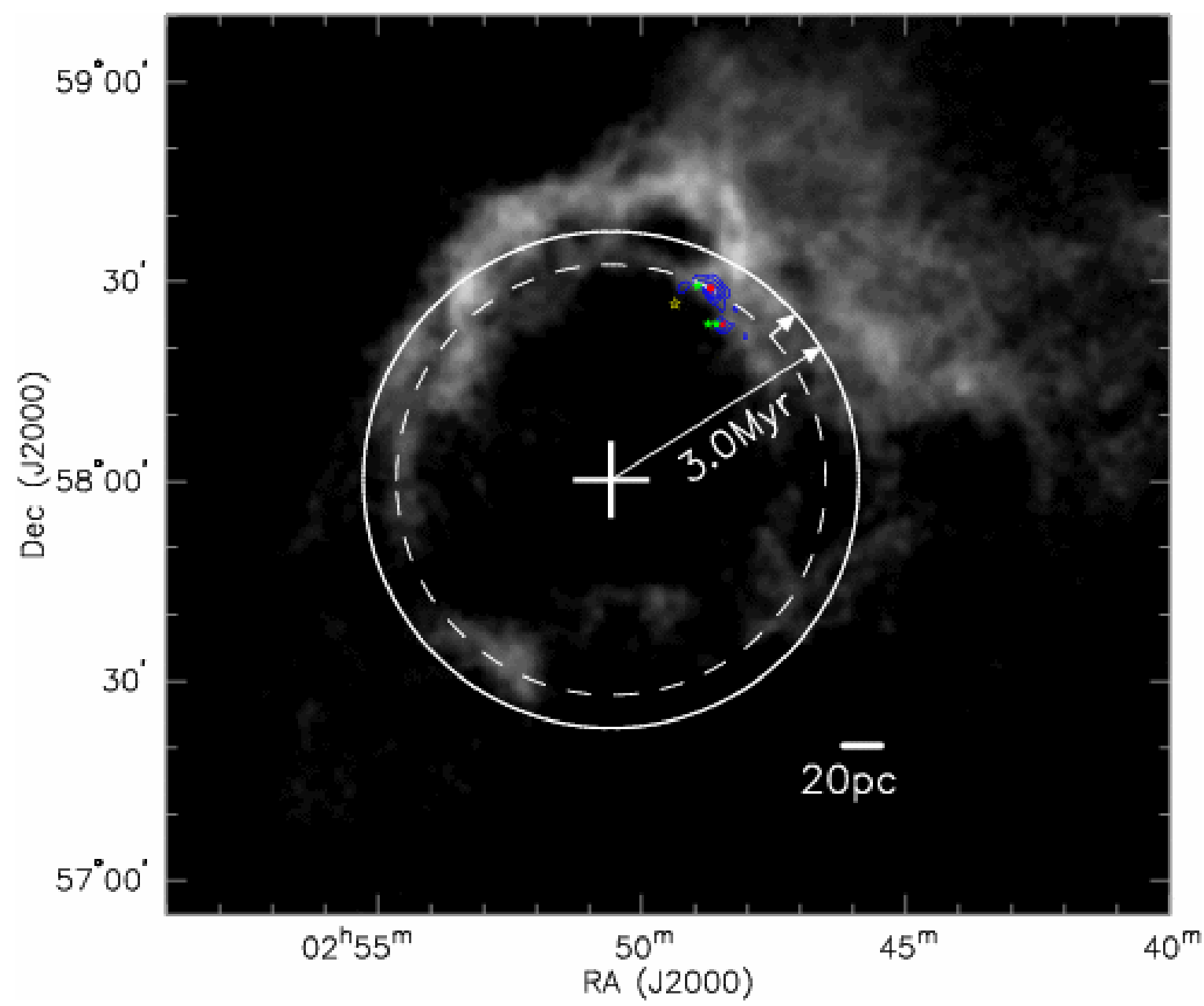

FIG. 6. - The location of Cloud 2 and the star clusters in the SNR shell GSH 138-01-94. The grayscale image and blue contours show the $\mathrm{H} \mathrm{I}$ and $\mathrm{CO}$ in $v_{\mathrm{LSR}}=-94$ to $-105 \mathrm{~km} \mathrm{~s}^{-1}$. The filled red circles show the location of the young embedded clusters. The solid circle shows the current location of the SNR shell with an estimated age of 3.0 Myr (see §4.2). The dashed circle shows the location of the Cloud $2-\mathrm{N}$ cluster. The small arrow shows the projected separation of 300 arcsec, from which the lifetime of the cluster can be estimated as 0.4 Myr (see $\S 4.2$ for detail). H I and CO data are same as that for Fig. 5 


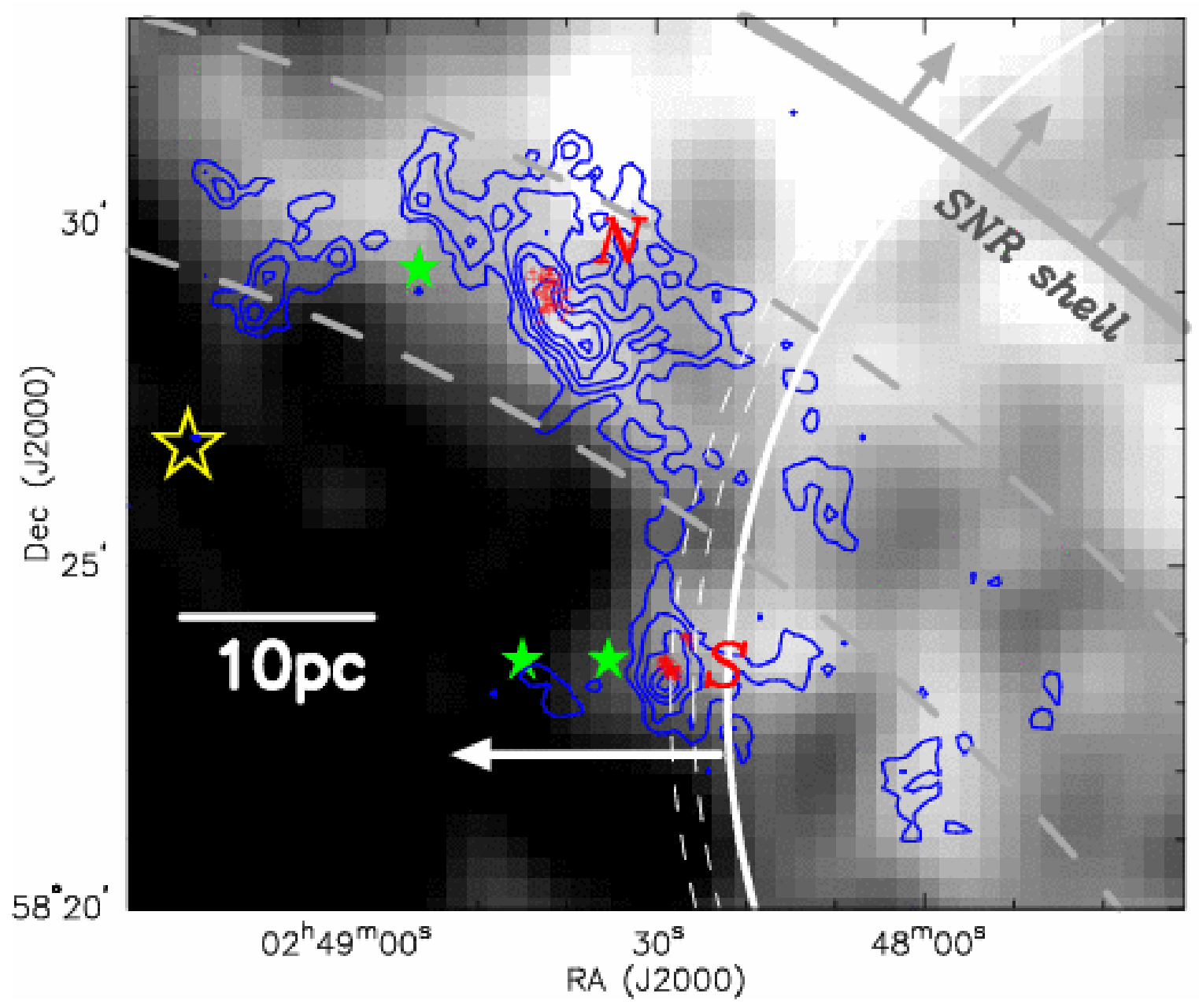

FIG. 7. - The relationship of the H I shells, Cloud 2 (blue contours), and the embedded clusters (red crosses). The outer edge of the SNR shell, GHS 138-01-94, is a thick gray curve, while the edge of the Cavity 2B (see the main text) is a thick white curve. Cloud 2-N is distributed between the two dashed gray curves whereas Cloud 2-S appears to be pushed back to inside the shell by the expansion of the Cavity 2B. The cluster-mode star formation in Cloud 2-S occurs at the shock front facing Cavity 2B. The H I and CO images are the same as for Fig. 4 [Refer to the ApJ online PDF to see the details of the distribution of the cluster members] The open yellow star mark and filled green star marks show the locations of the visible early-type star MR-1 and the bright NIR sources, respectively. 


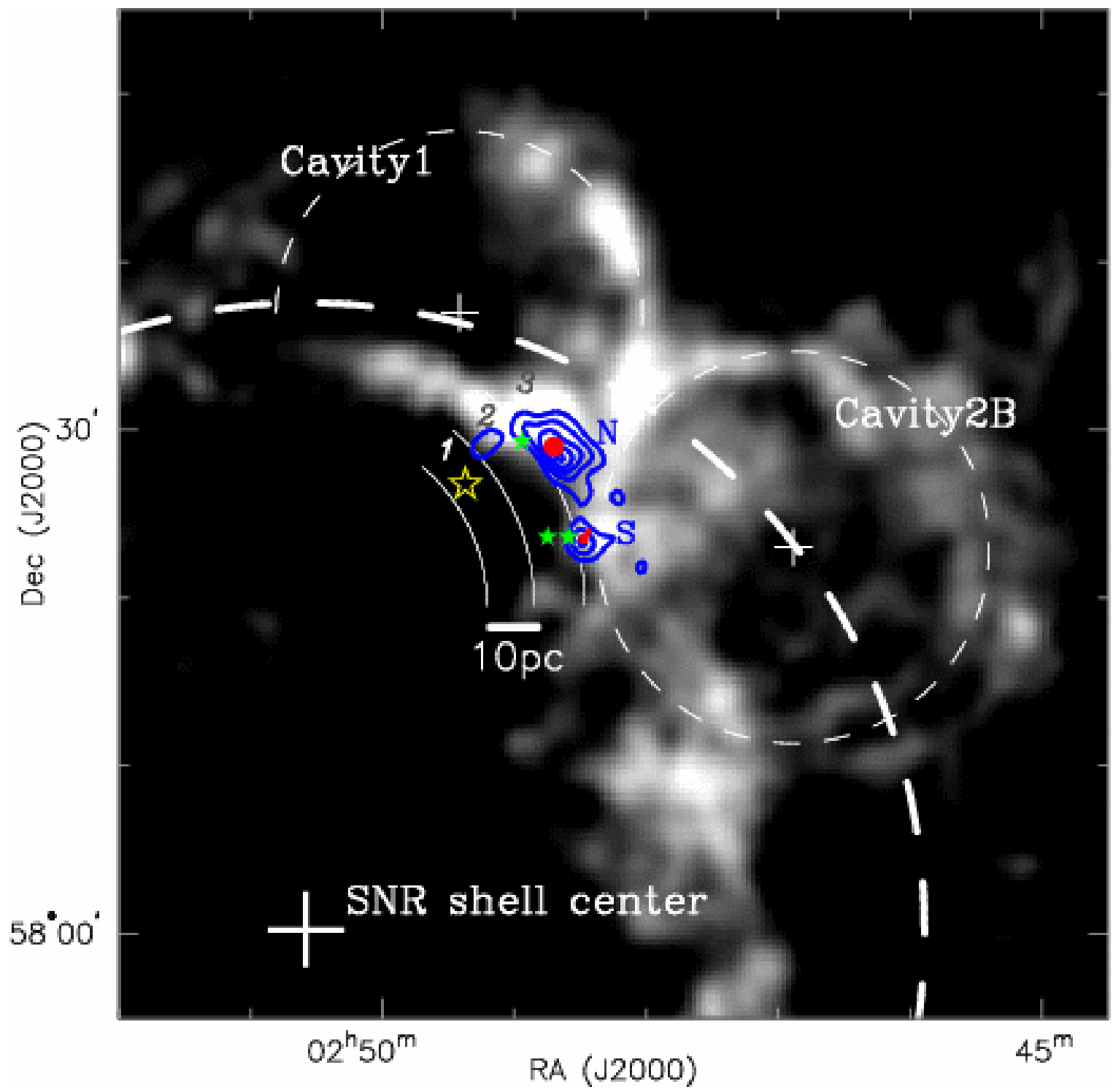

FIG. 8. - Cloud 2 and the associated members are shown in the H I $21 \mathrm{~cm}$ grayscale image. Cloud 2 is shown with ${ }^{12} \mathrm{CO}$ contours and its members are shown with a yellow open star (visible early-type star MR-1), filled green stars (bright NIR sources), and red circles (two embedded clusters). Progressive star formation from the inner side of the shell toward the edge (from zone 1 to 2 , then 3 ) is visible in this image. $\mathrm{CO}$ data is same as that for Figs. 1 and 4 except for the contour interval. The H I data from CGPS is integrated over a velocity range from -102 to $-105 \mathrm{~km} \mathrm{~s}^{-1}$, which shows the adjacent cavities clearly. 


\section{shell center}

$$
\begin{gathered}
\mathrm{t}=0 \\
-94.2 \mathrm{~km} / \mathrm{s}
\end{gathered}
$$
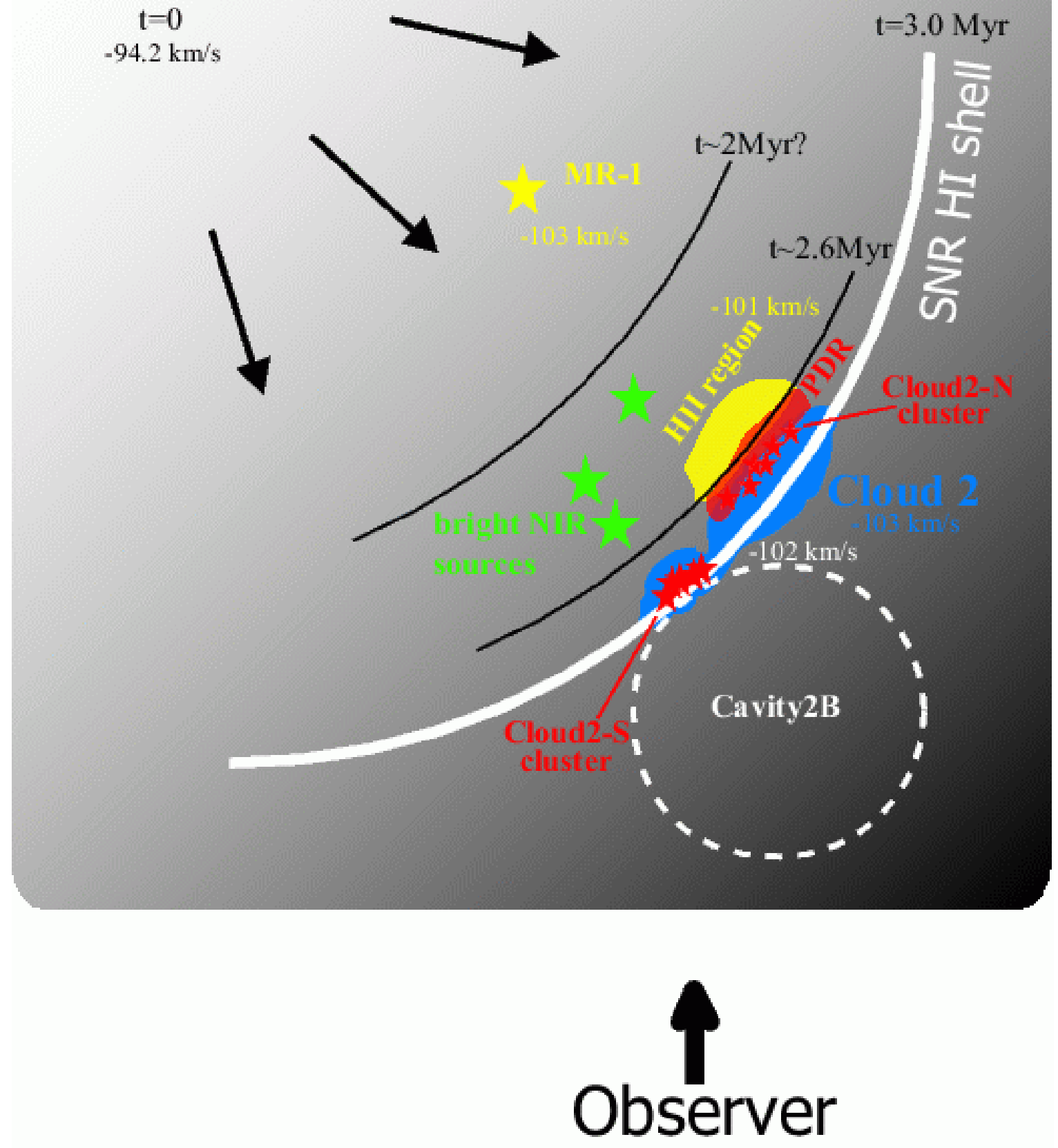

FIG. 9.- A schematic view of the progressive star formation by the SNR shell. The scales and the viewing angles are arbitrary. The times shown are counted from the time of the explosion of the SN. The radial velocities of the objects are shown (shell center, H I, and Cloud 2, Stil \& Irwin 2001; H II, de Geus et al. 1993; MR-1, Russeil et al. 2007). See the main text for the detailed discussion. 